\title{
Noninvasive follicular thyroid neoplasm with papillary-like nuclear features: a review for pathologists
}

Raja R Seethala, Zubair W Baloch, Justine A Barletta, Elham Khanafshar, Ozgur Mete, Peter M Sadow, Virginia A LiVolsi, Yuri E Nikiforov, Giovanni Tallini and

Lester DR Thompson

Department of Pathology, University of Pittsburgh Medical Center, Pittsburgh, PA, USA

\begin{abstract}
The rising incidence of papillary thyroid carcinoma is linked in part to inclusion of noninvasive follicular variant of papillary thyroid carcinoma. Despite its designation as carcinoma, noninvasive follicular variant of papillary thyroid carcinoma appears to be exceptionally indolent, often over treated by current treatment practices. Additionally, criteria for diagnosis have historically been subjective and challenging. Recently, an international multidisciplinary collaborative group performed a clinicopathologic survey of such cases with extended followup and concluded based on the outcome data that a revision in nomenclature was warranted, proposing 'Noninvasive Follicular Thyroid Neoplasm with Papillary-like Nuclear Features (NIFTP).' This monograph is a synopsis and guide for pathologists on NIFTP and focuses on histologic features, including inclusion and exclusion criteria used to define NIFTP, as well as grossing guidelines, reporting practices, and potential diagnostic limitations.

Modern Pathology (2018) 31, 39-55; doi:10.1038/modpathol.2017.130; published online 20 October 2017
\end{abstract}

The rising incidence of papillary thyroid carcinoma over the past several decades is due to many different factors. ${ }^{1}$ In addition to more vigilant clinical surveillance leading to detection of subcentimeter tumors, the increase is also partly attributable to the evolution of histologic criteria for the diagnosis of certain variants, most notably the follicular variant of papillary thyroid carcinoma. ${ }^{2}$ This diagnosis, from its inception, has been a diagnostic challenge for pathologists and a significant source of confusion and anxiety. The source of this difficulty lies in the subtlety and subjectivity of the diagnosis in a considerable number of cases. ${ }^{3}$ Further, emerging data regarding the indolent nature of a subset of follicular variant of papillary thyroid carcinoma that are encapsulated and/or noninvasive,${ }^{4-6}$ counterintuitive to their designation as 'cancer,' has added another layer of complexity to this problem, contributing to the broader problem of overdiagnosis and overtreatment of patients.

Correspondence: Dr Raja Seethala, MD, Department of Pathology, University of Pittsburgh Medical Center, A614.X Presbyterian University Hospital, 200 Lothrop Street, Pittsburgh, PA 15217, USA.

E-mail: seethalarr@upmc.edu

Received 2 February 2017; revised 24 August 2017; accepted 26 August 2017; published online 20 October 2017
Recently, an international multidisciplinary collaborative group performed an extensive clinicopathologic survey of 268 new cases extended follow-up to establish the specific subgroup of encapsulated/ noninvasive follicular variant of papillary thyroid carcinoma that may merit reclassification to a more appropriate nomenclature based on the outcome data. $^{7}$ With patient and clinician input, the consensus designation for this rigorously defined group of tumors is 'noninvasive follicular thyroid neoplasm with papillary-like nuclear features' (NIFTP), previously 'noninvasive follicular variant of papillary thyroid carcinoma.' The goal of this review is to provide a pathologist's perspective on NIFTP as well as a 'how-to guide' for the diagnosis of NIFTP.

\section{Historical perspective}

Papillary thyroid carcinoma and follicular thyroid carcinoma have been diagnostically distinct for many years. But prior to the 1970s, the distinction between papillary thyroid carcinoma vs follicular thyroid carcinoma was predicated on the predominant (ie, $>50 \%$ ) tumor growth pattern. Thus, if the tumor at low-power microscopic examination was mostly follicular in pattern, it was diagnosed as follicular thyroid carcinoma. ${ }^{8}$ Initial reports of follicular patterned 
tumors with nuclear features of papillary thyroid carcinoma date back to $1960,{ }^{9}$ but it was in the mid1970s that the specific term 'follicular variant of papillary thyroid carcinoma,' was first used to describe such tumors, ${ }^{10}$ signaling a move toward giving nuclear morphology precedence over growth pattern in defining papillary thyroid carcinoma. As of 2004, the most recent World Health Organization Classification of Endocrine Tumours book ${ }^{11}$ defined papillary thyroid carcinoma as a 'malignant tumor of the thyroid gland with a distinctive set of nuclear features' and has defined follicular thyroid carcinoma as 'a malignant tumor of the thyroid not having those nuclear features.' This shift in definition was acceptable as it allowed for the cytopathologic diagnosis of papillary thyroid carcinoma. However, the histologic diagnosis of follicular variant of papillary thyroid carcinoma raised several issues.

Characterization of follicular variant of papillary thyroid carcinoma as a variant of papillary thyroid carcinoma was expected to reflect a biologic behavior that would be more similar to classic papillary thyroid carcinoma, with a propensity for lymphatic and regional lymph node spread as opposed to the hematogenous spread and distant metastases seen in follicular thyroid carcinoma. However, this was not uniformly the case. Over the ensuing decades after the initial description, two major subtypes of follicular variant of papillary thyroid carcinoma were characterized: infiltrative follicular variant of papillary thyroid carcinoma and encapsulated follicular variant of papillary thyroid carcinoma. ${ }^{4,12}$ Infiltrative follicular variant of papillary thyroid carcinoma did indeed tend to behave more like classical papillary thyroid carcinoma ${ }^{4}$ with a similar propensity for nodal spread. However encapsulated follicular variant of papillary thyroid carcinoma still seemed to behave clinically more like a follicular neoplasm. When invasive, they tended to show tumoral capsular and vascular invasion with distant metastatic potential akin to follicular thyroid carcinoma, and when noninvasive, it was noted that these tumors behave indolently, essentially in a benign fashion much like follicular adenoma. This distinctive clinical behavior is also mirrored more recently by molecular findings. Infiltrative follicular variant of papillary thyroid carcinoma has a molecular profile that more resembles classical papillary thyroid carcinoma, while encapsulated follicular variant of papillary thyroid carcinoma has a molecular profile more similar to that of follicular adenoma/follicular thyroid carcinoma. ${ }^{5}$

These inconsistencies in encapsulated follicular variant of papillary thyroid carcinoma have fueled considerable controversy, mostly focused around tumors that showed no invasion. Firstly, the nuclear features, unlike classical papillary thyroid carcinoma, in the encapsulated follicular variant of papillary thyroid carcinoma tended to be more subtle and prone to subjective interpretation, resulting in considerable inter-observer variability about their presence or absence. ${ }^{13}$ Secondly, like follicular thyroid carcinoma, there were challenges in defining invasion in encapsulated follicular variant of papillary thyroid carcinoma (often compounded by post fine needle aspiration 'pseudo-invasion').,13-15 Given the biologic indolence of encapsulated follicular variant of papillary thyroid carcinoma without invasion, a number of alternative diagnostic terms were proposed: 'atypical adenoma', 'borderline follicular tumor', 'well differentiated thyroid tumor of uncertain malignant potential,' although the most commonly used term in the United States remained 'encapsulated follicular variant of papillary thyroid carcinoma.'16-19 Throughout these developments, endocrinologists, oncologists, and surgeons were conflicted about what constituted the most appropriate treatment recommendations for post-surgical patients: no additional treatment with watchful waiting; completion thyroidectomy (in patients with only a lobectomy); with or without radioactive iodine treatment. The financial, medical, and psychological impact would plainly vary considerably.

As more studies emerged documenting the indolent behavior of tumors currently designated as encapsulated follicular variant of papillary thyroid carcinoma, it became increasingly apparent that using the term 'cancer' to describe these lesions was not facilitating the clinical management decisions. These issues, along with such external catalysts as a National Cancer Institute recommendation in 2012 to revise terminology for select indolently behaving cancers (including thyroid), ${ }^{20}$ prompted the formation of an international multidisciplinary collaborative group to address this issue, which ultimately led to the reclassification of this tumor to NIFTP.

\section{Diagnosis of NIFTP}

\section{Inclusion Criteria}

Growth pattern. Put succinctly, inclusion criteria for NIFTP are essentially those used to previously characterize noninvasive encapsulated follicular variant of papillary thyroid carcinoma. ${ }^{17,21}$ All such tumors are required to be well demarcated, showing a discrete interface with the surrounding thyroid parenchyma. NIFTP encompasses tumors that have a well-defined complete capsule with smooth musclewalled vessels, those that are partly encapsulated, and those that are unencapsulated but are still well delineated from adjacent parenchyma (Figures 1 and 2). ${ }^{6}$ Tumors may be cystic and have a mixture of macro and microfollicular growth. It is not uncommon for microfollicular foci to intercalate between more attenuated macrofollicular areas in NIFTP (Figure 3a).

Cytomorphonuclear features. Importantly, as before the terminology revision, these tumors must 

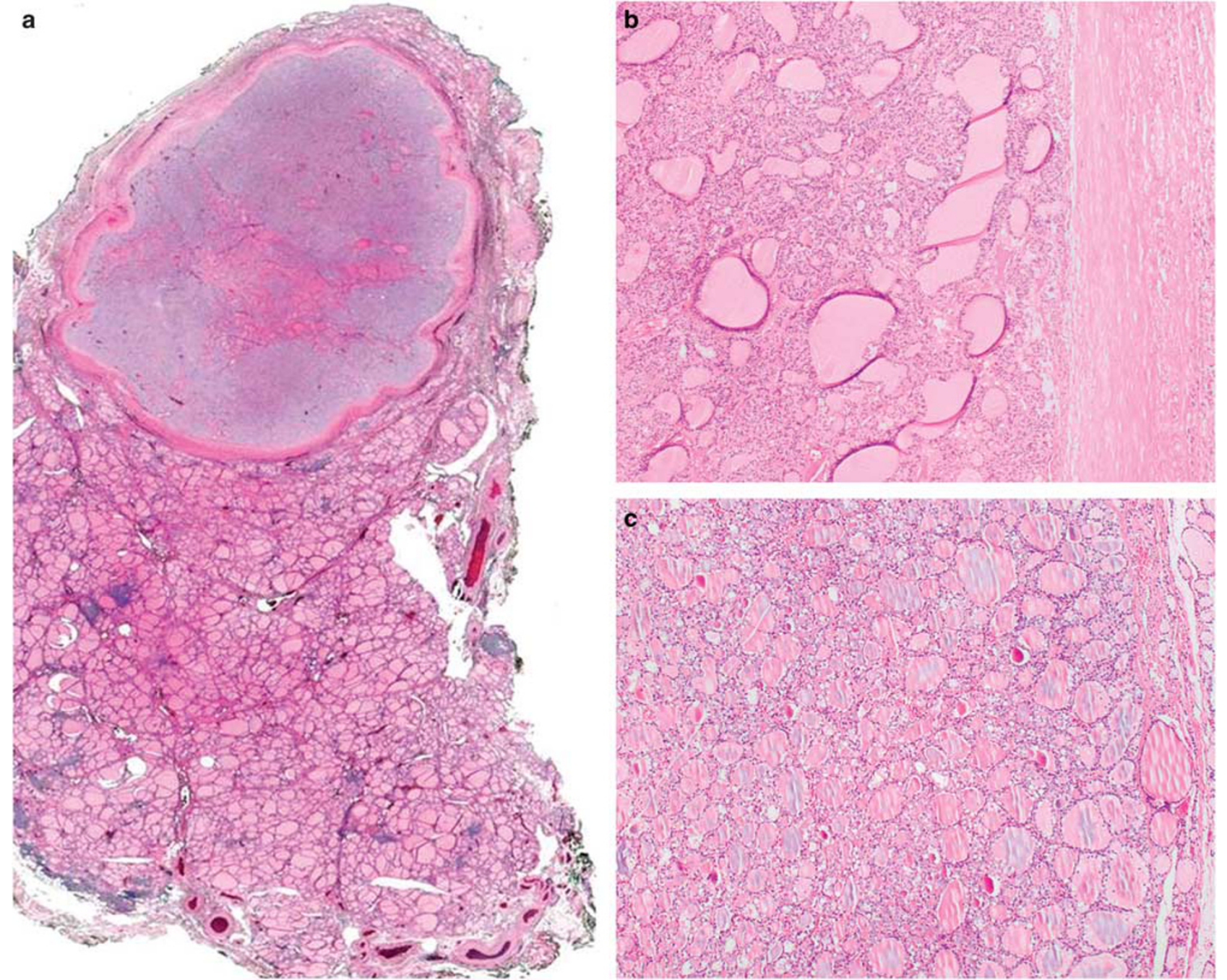

Figure 1 (a) At scanning magnification, this NIFTP is sharply demarcated from the adjacent thyroid by a well-formed sclerotic capsule. While follicles with dense colloid are visible even at this magnification in the center, most of the tumor has a distinctive light gray blue cast, attributable to the papillary thyroid carcinoma-like nuclear clearing common to these tumors. (b) Some tumors have a thick fibrous capsule, (c) while other tumors have a thin capsule.

still have nuclear features that would be seen in papillary thyroid carcinoma. Unlike classical papillary thyroid carcinoma, NIFTP nuclear features are typically more subtle, and range from diffuse in distribution to patchy and multifocal (so-called sprinkling sign), usually more prominent in the more cellular, microfollicular areas (Figure 3a) as well as being accentuated at the periphery of the neoplasm near the capsule (if one is present) (Figure 3b). NIFTP may show varying degrees of intratumoral fibrosis, and the colloid may range from dense or hypereosinophilic (usually in more microfollicular areas) to watery (usually in more macrofollicular or cystic areas).

\section{Exclusion Criteria}

Since NIFTP is no longer classified as a carcinoma, strict exclusion criteria were adopted to ensure that NIFTP retains a consistently indolent behavior, thus facilitating meaningful clinical management approaches. The diagnosis of NIFTP is only established when both inclusion and exclusion criteria are met. The following are a list of such criteria, that when identified, would exclude the use of NIFTP.

Invasion. Invasion beyond the border of a tumor precludes a diagnosis of NIFTP. For encapsulated tumors, this entails complete tumor capsule penetration, similar to what would be required to diagnose invasion in follicular thyroid carcinoma (Figure 4a and b). For well-circumscribed tumors lacking a fibrous capsule, infiltration of tumor cells into adjacent uninvolved parenchyma is adequate and may not have the tell-tale 'mushroom like' configuration expected in tumor capsule penetration. Also precluding this diagnosis are lymphatic and/or vascular invasion (Figure 4c). This is defined by tumor cells within an endothelial lined space in the capsule or in vessels outside the tumor, often 

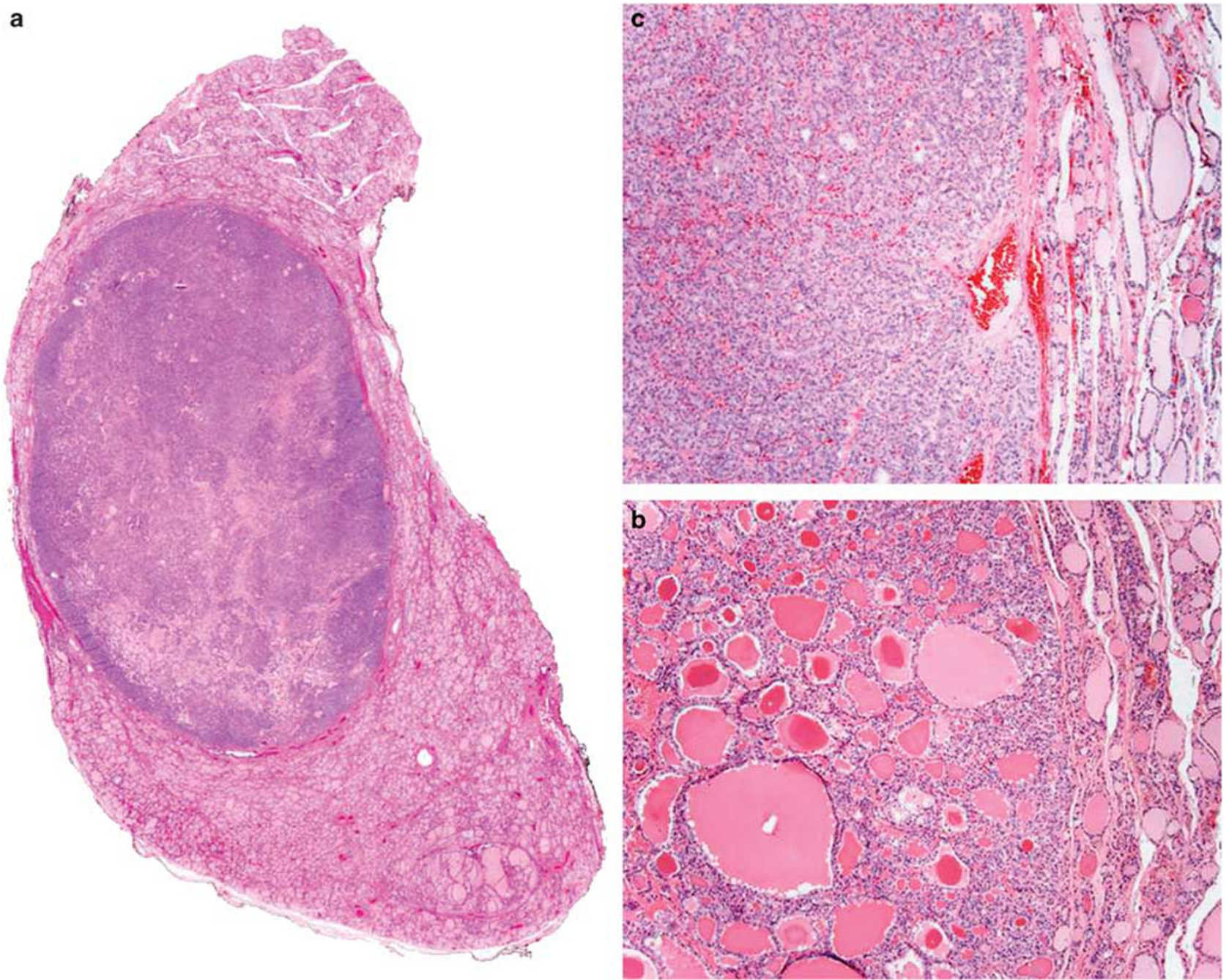

Figure 2 (a) This NIFTP shows a rounded contour at scanning magnification, but does not have a distinct capsule. (b) This tumor has a thin partial capsule. (c) This tumor entirely lacks a capsule, but has a sharp interface between tumor and benign parenchyma.

accompanied by thrombus or showing wall attachment (ie, not free floating tumor). ${ }^{22}$ Of course, perineural invasion and extrathyroidal extension also preclude NIFTP. In the rare scenario when the tumor capsule is surgically transected, and thus the tumor is at the margin but not necessarily extrathyroidal in extent, NIFTP terminology is still not recommended. Because a diagnosis of NIFTP requires a lack invasion to render the diagnosis confidently, adequate, meaning complete, sampling of the tumor-to-normal interface is advocated (see below).

Pattern of growth. By definition, the tumor must show a predominantly follicular architecture. Occasional tumors may show a small component of solid, trabecular, or insular architecture (Figure 5). However, these other patterns of growth cannot exceed $30 \%$ of the overall tumor volume. When these patterns are present, very careful review is required to exclude progression to poorly differentiated carcinoma. ${ }^{5,23}$ The presence of other specific morphologies, such as tall cell features, columnar cell features, or cribriform architecture would all exclude the use of NIFTP. If there is difficulty in interpreting the pattern of growth, then NIFTP should not be the diagnosis applied.

Papillary structures. Initially, single, isolated papillae, comprising $<1 \%$ of the tumor volume were acceptable for NIFTP classification. However, this designation is admittedly arbitrary. The allowance for rare papillary structures in NIFTP was done for principally practical purposes, as sectioning artifacts and focal changes should not result in an overly restrictive diagnostic category. In practical terms, even occasional well-developed papillary structures (Figure 6a) should warrant further scrutiny for conventional areas. In fact, a recent study advocates for an even stricter approach noting that even with noninvasive tumors with $<1 \%$ papillae, a small subset may show nodal metastases, and BRAF V600E mutations, the latter which should not be seen in NIFTP-EFVPTC spectrum of tumors (see below). On 

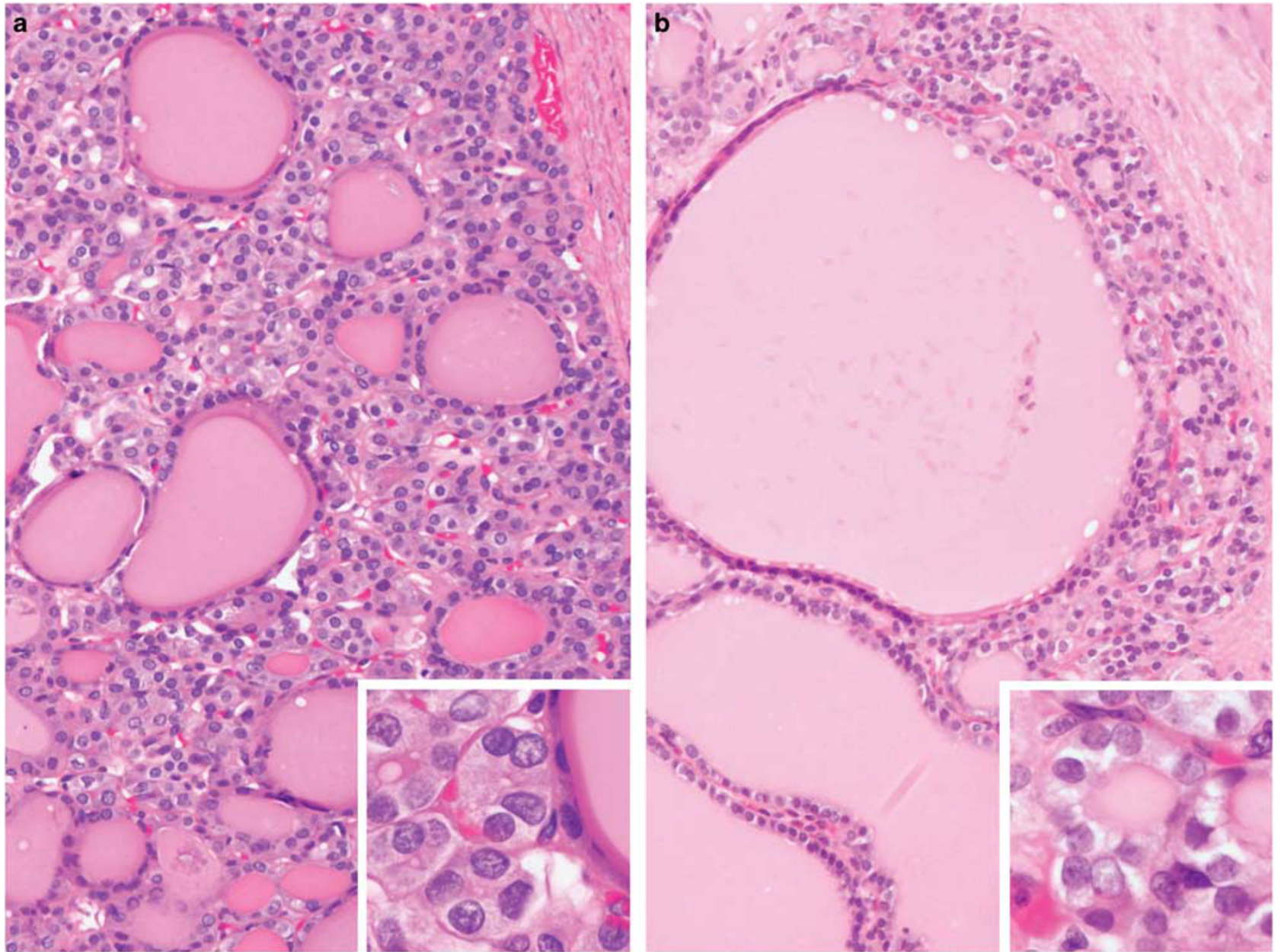

Figure 3 (a) NIFTP often demonstrates microfollicles intercalating between attenuated larger follicles as seen here in this encapsulated tumor. Inset-diagnostic nuclear features are more common in microfollicular areas. (b) NIFTP also frequently shows accentuation of nuclear features at the periphery, as evidenced by the paler appearance at low-power magnification. Inset-the periphery demonstrates readily visible diagnostic nuclear features. In both insets, the nuclear features are not as prominent as commonly seen in classic papillary thyroid carcinoma.

the other hand, when no papillae are accepted the NIFTP categorization becomes 'cleaner.' 24 It is now recommended that there should be NO well-formed papillae within the NIFTP spectrum. By definition, a papillary structure represents a fibrovascular core lined by neoplastic follicular cells. Fibrovascular cores should be confirmed histologically, with delicate endothelial cells surrounding erythrocytes. Sanderson polsters, which are collections of small follicles that project into the lumen of follicles, but lack true fibrovascular cores (Figure 6b) do not qualify as true papillae.

Psammoma bodies. Psammoma bodies in the context of papillary thyroid carcinoma represent mummified and dead papillae that have undergone concentric lamination by calcium. ${ }^{25}$ Therefore, the presence of psammoma bodies would suggest papillae, which by definition should be extremely rare in NIFTP. Furthermore, identification of a psammoma body in the fibrous septae of the parenchyma within lymphatics or within lymph nodes, is consistent with lymphatic invasion. In the absence of another plausible explanation for psammoma bodies in lymphatics (ie, a concurrent classical papillary thyroid carcinoma), this should be taken as evidence of invasion, thus disqualifying the tumor from the NIFTP category.

Tumor necrosis. Any areas of 'true' tumor necrosis, identified as comedonecrosis or confluent necrosis, would exclude the use of NIFTP, as these are highgrade features. Ghost outlines of the neoplastic cells will frequently be seen (Figure 7a), helping to confirm true necrosis. ${ }^{5,23}$ Experience with encapsulated tumor with high-grade nuclear features is limited, and though most of the rare cases reported have a favorable behavior, one recent case without tumoral capsular or lymphovascular invasion has been reported with metastasis. ${ }^{26}$

Increased mitoses. Akin to necrosis, the identification of increased mitoses, which is defined as more than 3 mitoses per 10 high-power fields using a 

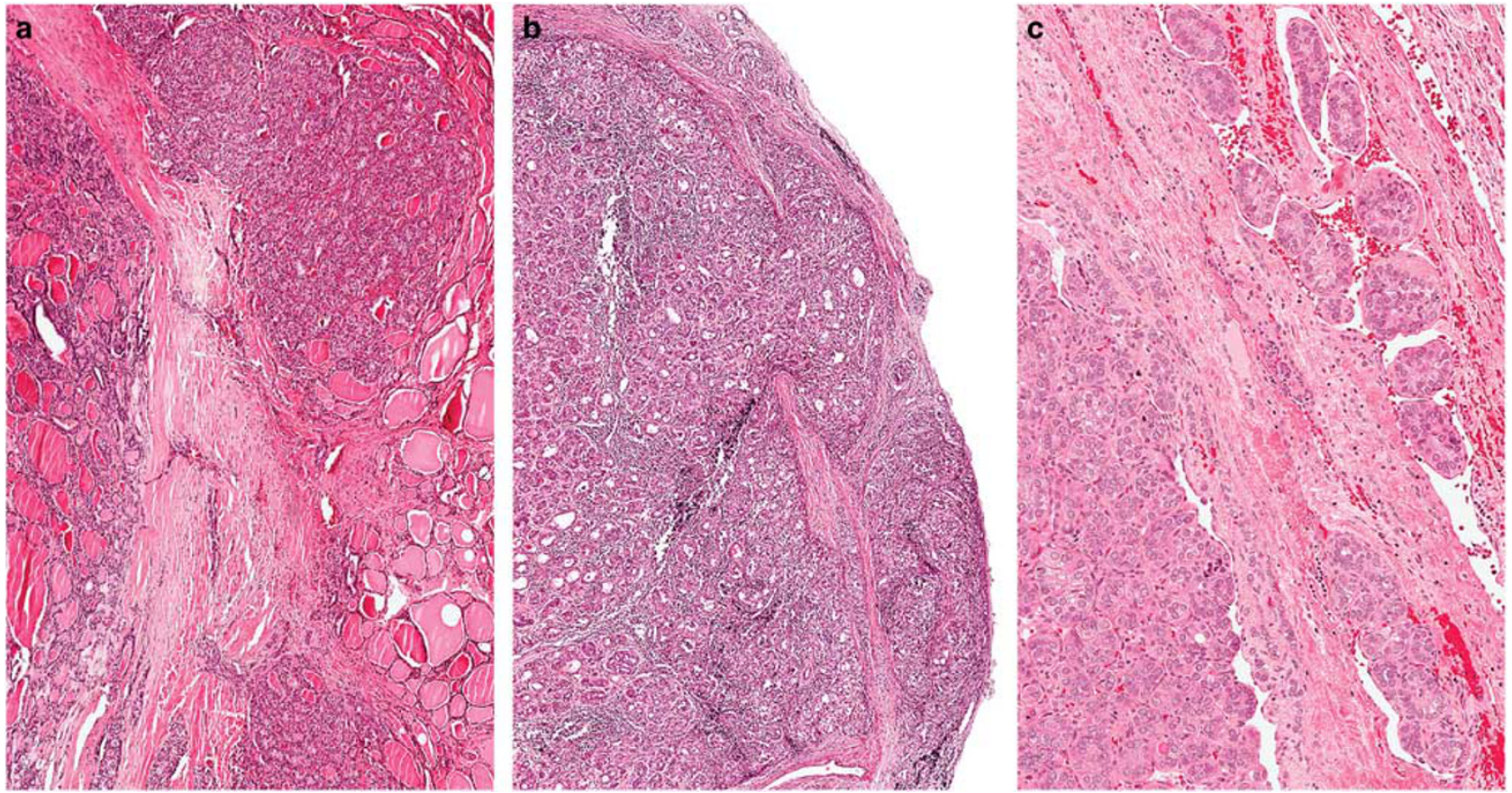

Figure 4 Capsular invasion, precluding the diagnosis of NIFTP. (a) Tumor has expanded through the capsule into the adjacent parenchyma. (b) The capsule is invaded by the neoplastic cells, forming a mushroom-shaped invasive front. (c) Lymphovascular invasion by neoplastic cells, excluding the diagnosis of NIFTP. There is associated hemorrhage and tumor thrombi that are attached to the vessel wall.

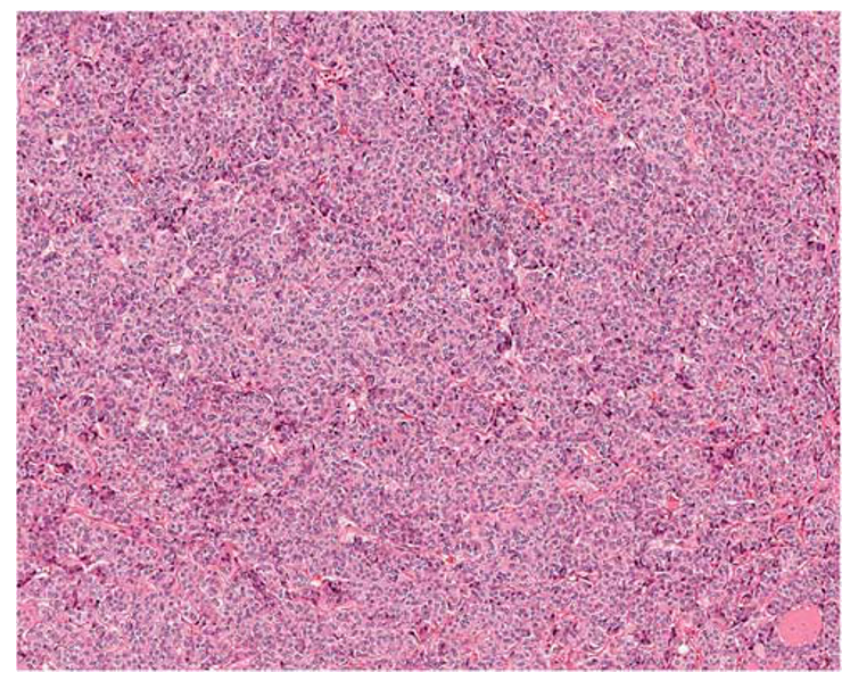

Figure 5 A solid pattern in a papillary carcinoma (note colloid in the lower right corner, arrow). This pattern cannot exceed $30 \%$ for a NIFTP diagnosis.

standard $\times 40$ magnification and $\mathrm{a} \times 10$ objective lens ( $\times 400$ total magnification), is generally considered a high-grade feature when used in conjunction with solid, trabecular, and insular growth, and necrosis, ${ }^{5,23}$ and would disallow the use of NIFTP. The intent however, is not to use this criterion without an appropriate morphologic context, since even hyperplastic nodules may show foci of mitotic activity. ${ }^{27}$ Thus mitotic counts as a marker of progression beyond NIFTP are most appropriately determined in cellular/microfollicular patterned foci away from a fine needle aspiration tract or needle biopsy site, as it is not uncommon to have mitoses in these areas. Additionally, 10 consecutive high-power fields are recommended for evaluation rather than counting 'hot spots,' which may be prone to inflate mitotic indices. Elevated mitotic counts are usually, but not always, accompanied by solid, trabecular, or insular growth, occasionally in the form of a nodule within a broader follicular patterned nodule (Figure $7 b-d)$.

Multifocality and size. It is important to note that tumor multifocality may be seen with any thyroid gland tumor. Therefore, the diagnosis of NIFTP does not and should not exclude the diagnosis of another topographically separate tumor in the gland. However, for staging purposes, NIFTP is not included, as it is only the malignant tumors that would be incorporated into the staging. At present, tumors below $1 \mathrm{~cm}$ and multiple NIFTP within the same thyroid have not been addressed in a methodical fashion, although limited data suggest that they are acceptable for inclusion; ${ }^{28}$ further evaluation is required.

\section{Nuclear scoring system: revisiting the core diagnostic feature}

Although NIFTP is established based on a constellation of architectural, stromal, and nuclear characteristics, it is the latter by which this entity is ultimately 

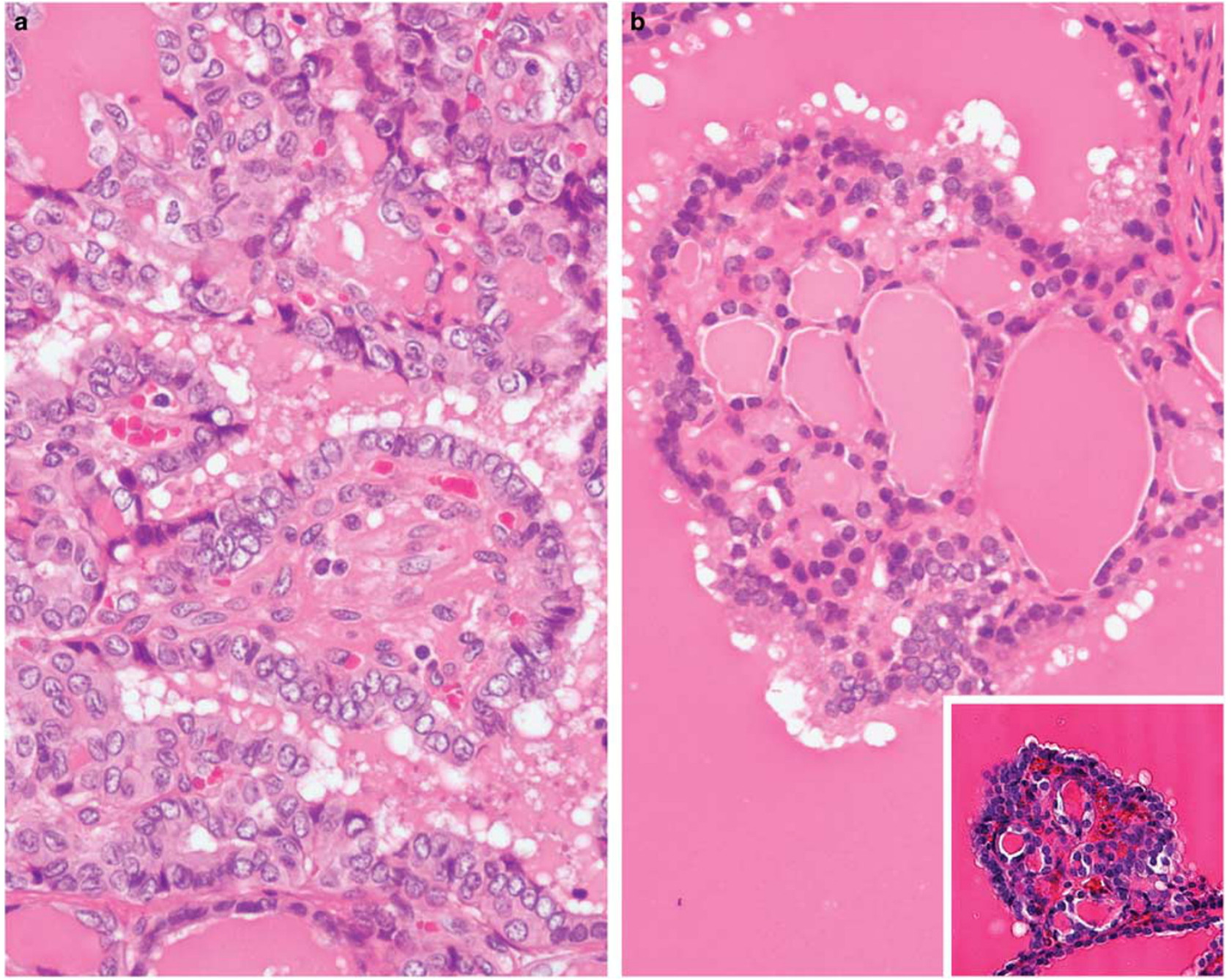

Figure 6 (a) Despite a predominant follicular pattern, there is a well-developed papilla with a fibrovascular core in the center of this classic papillary thyroid carcinoma. (b) This NIFTP shows a pseudopapillary structure, with follicles rather than vessels within the connective tissue core, recapitulating the features of the Sanderson polster seen in this hyperplastic nodule (inset).

defined. The nuclear features of NIFTP are essentially those of papillary thyroid carcinoma. ${ }^{29}$ These include the familiar list of features deemed important for the diagnosis of papillary thyroid carcinoma: nuclear enlargement, overlap, elongation, irregular nuclear membranes, grooves, nuclear indentations or folds, intranuclear cytoplasmic inclusions, powdery chromatin, and chromatin clearing. ${ }^{13}$ It is critical that these criteria are not derived in isolation, but should be compared to the adjacent normal, since background disease (ie, thyroiditis), slide preparation and processing artifacts may induce some baseline variation in nuclear size and chromatin appearance.

The threshold for sufficient nuclear features for its forerunner, follicular variant of papillary thyroid carcinoma, has been a point of contention, both in the literature ${ }^{30-32}$ and in day-to-day practice. Among experts, reproducibility for this category has been described as mediocre at best, and demonstrating considerable national geographic variation in threshold for diagnosis. ${ }^{3,13,14}$ The working group decided that in addition to defining the clinical and histologic features of NIFTP, a secondary objective would be to improve the reproducibility of criteria and establish more detailed guidelines to define what constitutes adequate and minimum nuclear features for a NIFTP diagnosis.

This resulted in the development of a simple scoring system. In order to simplify assessment of the nuclear features of follicular patterned lesions, characteristics were grouped into broader categories: (i) size and shape (enlargement, elongation, and overlap; Figure 8a), (ii) membrane irregularities (grooves, folds, irregular contours, pseudoinclusions; Figure 8b); and (iii) chromatin characteristics ('glassy' nuclei, powdery, delicate chromatin, chromatin margination to the membrane, and clearing; Figure 8c). If a given category of features is considered adequate in extent and quality, it is assigned one point. Two of three categories must show adequate features (ie, score 2 of 3 ) to allow for 

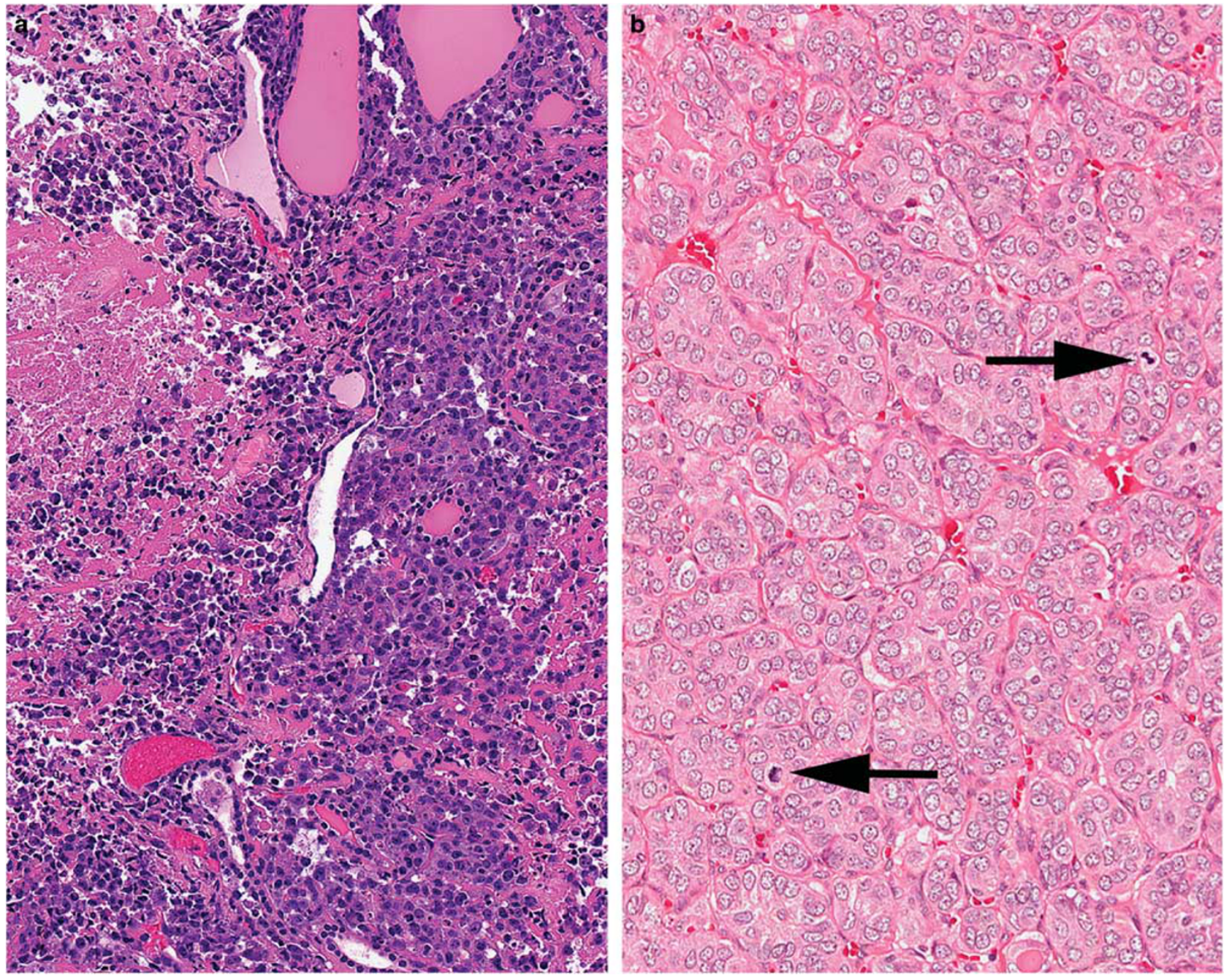

Figure 7 (a) In addition to the solid growth seen here, tumor necrosis excludes a diagnosis of NIFTP. (b) This area with trabecular architecture shows an increased number of mitoses (arrow), which if more than 3 mitoses per 10 high-power fields would exclude NIFTP.

the cytomorphonuclear diagnosis of NIFTP. Even within what is considered adequate, nuclear features range from subtle to obvious, reminiscent of those seen in classical papillary thyroid carcinoma. While not exclusionary, excessively overt nuclear features, even in an encapsulated/well-demarcated lesion should raise concern for a conventional papillary thyroid carcinoma with prominent follicular pattern, and thus further scrutiny is warranted before accepting the tumor as NIFTP. This may entail deeper levels, total submission for smaller nodules, or additional sections of the central portion for larger lesions (see below).

This scoring system is not mandatory to diagnose NIFTP in day to day practice, but it has advantages in facilitating the diagnosis. It was kept at a low level of complexity to ensure practicality, and in essence, the main effect of the scoring scheme is the categorization of the mental checklist that all pathologists go through to arrive at a diagnosis in a follicular patterned thyroid lesion. More importantly, this system has undergone an unprecedented statistically rigorous validation against an actual reference standard. While not perfect even with an extended panel of molecular studies, ${ }^{33}$ molecular status was the most reasonable standard, since outcome was uniformly favorable in this group of tumors and thus not discriminatory. Almost $80 \%$ of NIFTP demonstrate clonal alterations. As predicted, NIFTP are more similar to follicular adenoma and follicular thyroid carcinoma in that they frequently demonstrate RAS mutations with none demonstrating RET/ PTC (ie, RET-CCDC6/RET-NCOA4) or BRAF V600E mutations. Other mutations/fusions documented are also along the RAS like molecular spectrum and include: PAX8/PPARG and THADA fusions, and EIF1AX and BRAF K601E mutations.

Indeed, this simple scoring scheme showed 8594\% accuracy in predicting molecular alteration status, with a modest reproducibility (intraclass correlation coefficient). Lessons learned here were that historically, differing personal thresholds for what defined follicular variant papillary thyroid carcinoma were a cause of interobserver variability 


\section{a SIZE AND SHAPE \\ -Enlargement \\ -Elongation \\ -Overlapping}

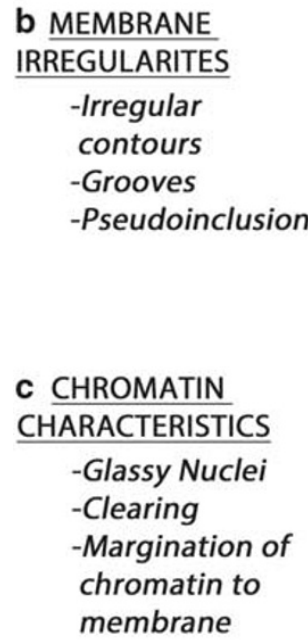

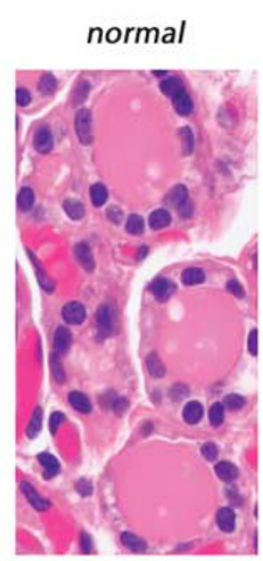
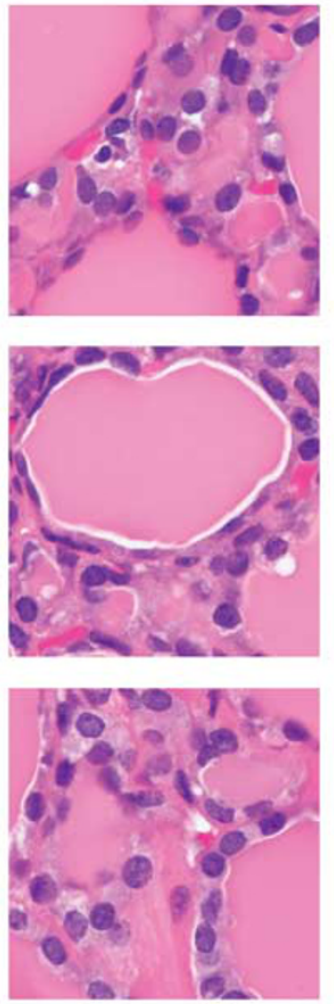

SUFFICIENT
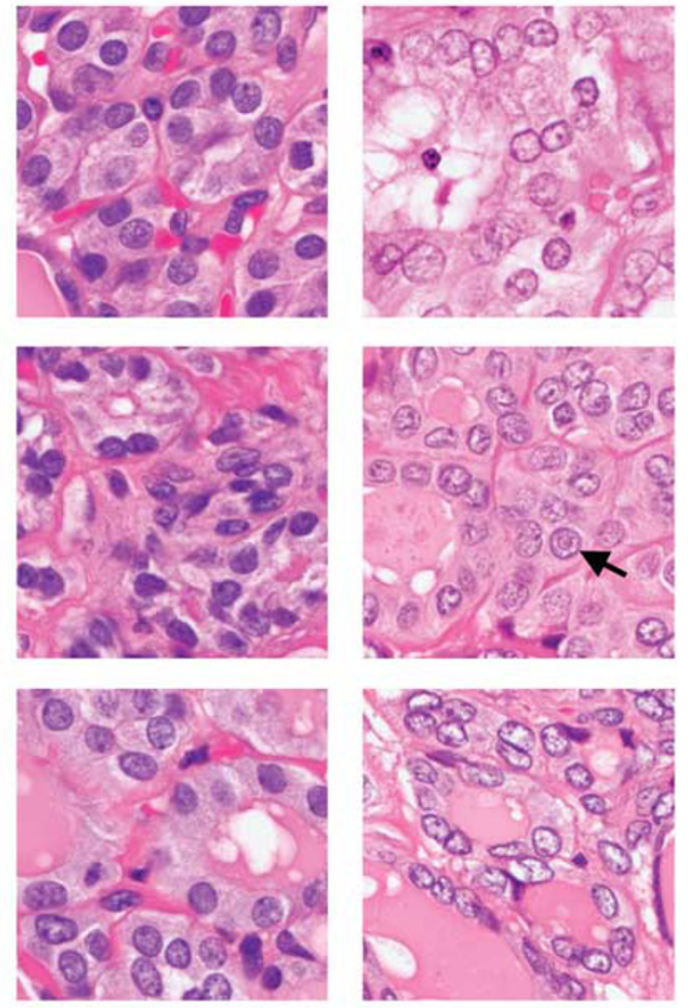

Figure 8 Nuclear scoring scheme for NIFTP divided into three broad categories: (a) size and shape, (b) membrane irregularities, and (c) chromatin characteristics. Nuclear features in a lesion should be compared to adjacent nonneoplastic parenchyma. Images to the left of the divider are insufficient for the diagnosis of NIFTP, while those to the right are sufficient. The images to the far right of the divider depict pronounced nuclear features (arrow-pseudoinclusion) that if highly prevalent in the lesion should warrant further scrutiny for exclusionary features of NIFTP.

in earlier literature. When simply asked whether a set of nuclear features is abnormal or not, pathologists with differing thresholds became more aligned.

Aside from extent of nuclear features, distribution of these features is often used to distinguish between NIFTP and follicular adenoma or even hyperplastic nodule. Distribution was considered in the construction of the scoring system. It was noted that the more diffuse the diagnostic nuclear features were in a lesion, the more likely it was to have a molecular alteration. However, statistically, the accuracy gain was minimal and not significant, and it was therefore not formally included in the scoring system. From a practical standpoint it is reasonable to still consider the extent of the nuclear features when assessing a follicular patterned nodule, and all cases with a molecular alteration in the test set demonstrated diagnostic nuclear features in more than $30 \%$ of the tumor volume, which is a reasonable rule of thumb. However, it cannot be a 'hard and fast' rule since cases with a 'sprinkling' of very overt nuclear features within an otherwise bland nodule would not conform to this rule, yet would still be acceptable for NIFTP (Figure 9). ${ }^{34}$

Table 1 summarizes a stepwise algorithm that can be used to arrive at a diagnosis through application of inclusion criteria, exclusion criteria, and nuclear score.

\section{Clinical behavior: the implications of $A$ NIFTP diagnosis}

As noted above, the very reason that NIFTP was defined was a concerted effort to curb overdiagnosis of at least one form of 'thyroid cancer,' specifically the noninvasive follicular variant of papillary thyroid carcinoma. In addition to more recent studies,, 28 a body of literature exists supporting that invasion rather than nuclear features correlates with the 
outcome of noninvasive follicular variant papillary thyroid carcinoma. The multi-institutional cohort study of over 100 patients that first defined NIFTP ${ }^{7}$ showed no recurrence or metastasis when followed for 10-26 years (median, 13 years). Additionally, a literature review of $\sim 600$ well-documented noninvasive follicular variant papillary thyroid carcinoma would also potentially qualify as NIFTP. To the best

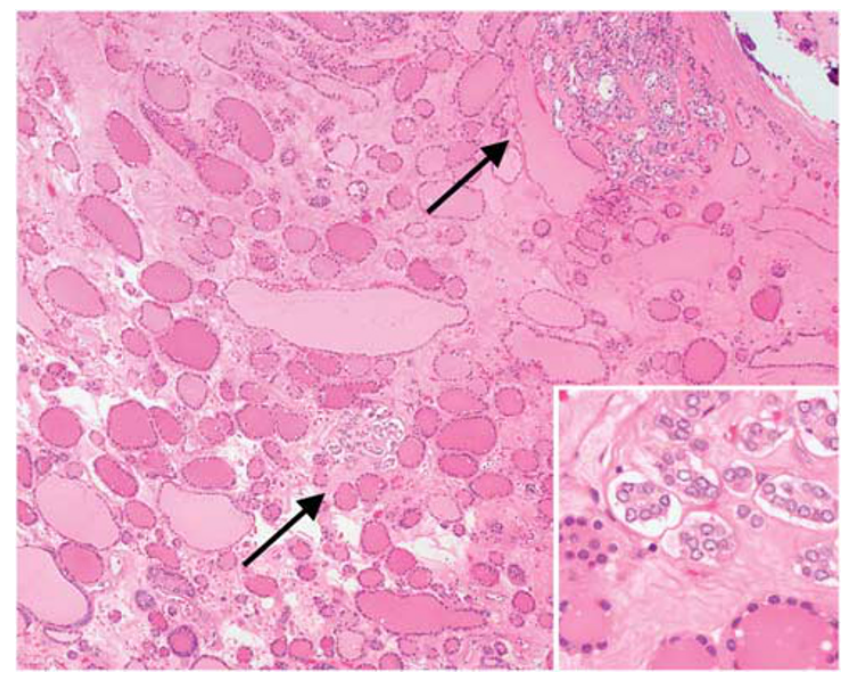

Figure 9 A rare tumor with a 'sprinkling' of small microfollicular foci (arrows) in an otherwise bland nodule. On closer examination (inset) demonstrate follicles that cleft from their surrounding basement membrane and show the nuclear features sufficient for inclusion as NIFTP. of our knowledge there are up to three recurrences/ distant metastases $(\sim 0.5 \%)$ from this cohort (Table 2). $4,6,7,24,28,35-41$ One of these two cases recurred at the thyroid bed in a tumor with a positive resection margin; the second case had incomplete sampling of the capsule, failing to confirm the noninvasive nature of the tumor. The recent case with reported distant metastasis is viewed with the caveat that it was a $0.6 \mathrm{~cm}$ tumor that harbored a BRAF V600E mutation. ${ }^{24}$ As per the study, the primary tumor technically fulfilled the $<1 \%$ papillae cutoff. However, this tumor would no longer be considered NIFTP using the stricter criteria for papillae that we advocate. However, it must also be noted that NIFTP criteria, particularly percentage cut-offs, are not well validated for subcentimeter nodules (see below). Additionally, this tumor shows sclerosis (Figure 6 in this study)—a marker of aggression in papillary microcarcinomas (which this case would have been considered historically). ${ }^{42} \mathrm{An}$ additional potential case of NIFTP from a retrospective study of 2978 thyroid tumors initially diagnosed as benign but with lymph node and lung metastasis was identified but was not well documented histologically. ${ }^{43,44}$ Another case with lymphnode metastasis was reported in a cytologic study of NIFTP, but without adequate histologic documentation. ${ }^{45,46}$ Cho et al ${ }^{24}$ identify a $3 \%$ nodal metastatic rate in NIFTP even when papillae are entirely excluded, however, despite the extensive examination by the authors, it is plausible that an undetected microcarcinoma is responsible for this

Table 1 An algorithm for the diagnosis of noninvasive follicular thyroid neoplasm with papillary-like nuclei (NIFTP)

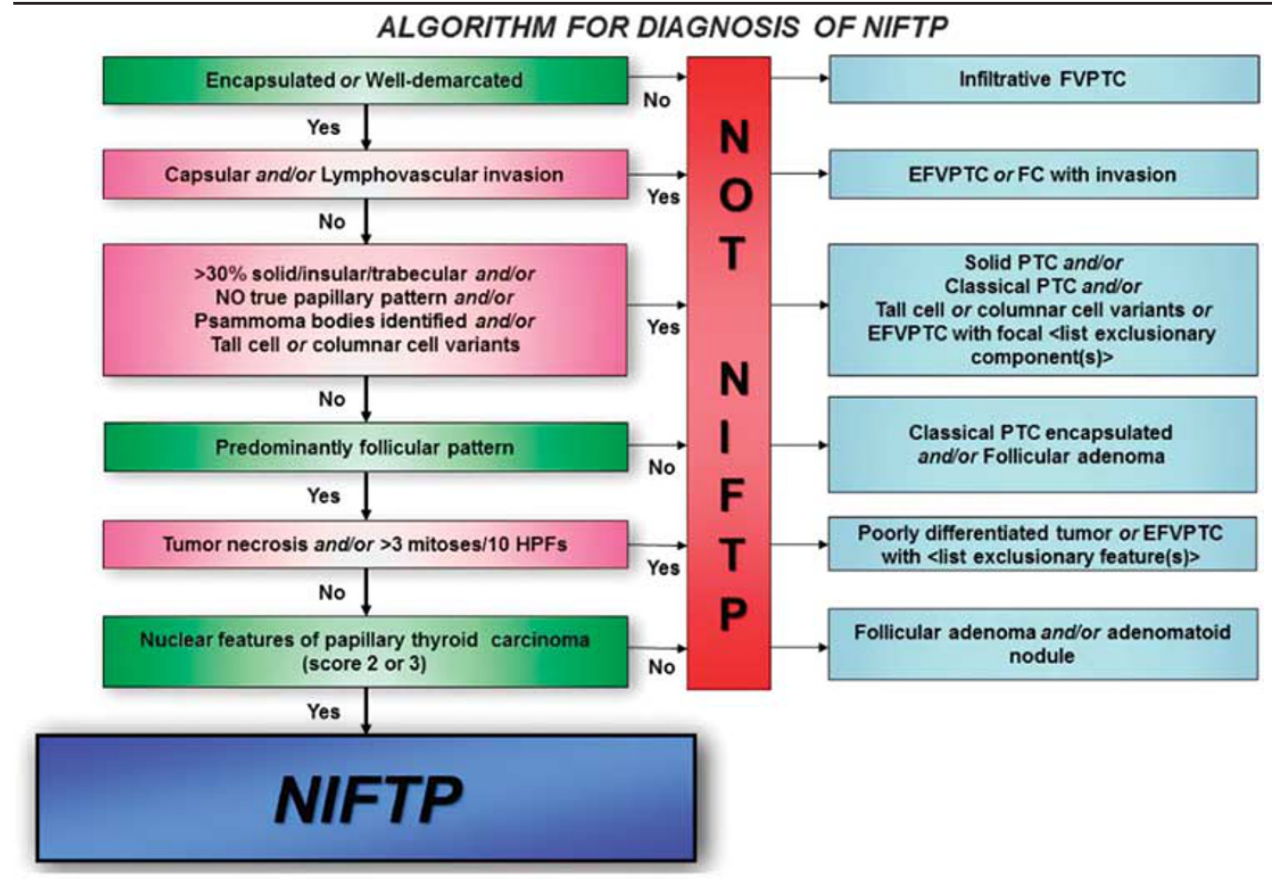

EFVPTC, encapsulated/noninvasive papillary thyroid carcinoma; FC, follicular carcinoma; FVPTC, follicular variant of papillary thyroid carcinoma; PTC, papillary thyroid carcinoma. 
Table 2 Clinicopathologic features of noninvasive follicular variant papillary thyroid carcinoma/NIFTP

\begin{tabular}{|c|c|c|c|c|c|c|}
\hline Reference & Follow-up & Type and number of FVPTC cases & $\begin{array}{l}\text { Invasion (without; capsular; } \\
\text { vascular) }\end{array}$ & $\begin{array}{l}\text { Lymph-node } \\
\text { metastasis }\end{array}$ & $\begin{array}{l}\text { Recurrence/distant } \\
\text { metastasis }\end{array}$ & $\begin{array}{c}\text { Tumor-related } \\
\text { death }\end{array}$ \\
\hline Baloch et al ${ }^{35}$ & $\mathrm{n} / \mathrm{r}$ & Encapsulated $N=5$ & $\begin{array}{l}1 \text { Capsular invasion } \\
3 \text { Vascular invasion } \\
1 \text { Multifocal papillary } \\
\text { carcinomas identified }\end{array}$ & $\mathrm{n} / \mathrm{r}$ & All 5-bone metastases ${ }^{a}$ & $\mathrm{n} / \mathrm{r}$ \\
\hline Liu et $a l^{4}$ & Median 11.1 yrs & $\begin{array}{l}\text { Encapsulated } N=61 \\
\text { Infiltrative } N=17\end{array}$ & $\begin{array}{l}\text { Without invasion } N=43 \\
\text { With invasion } N=18\end{array}$ & $\begin{array}{c}0 \\
3(17 \%) \\
11(65 \%)\end{array}$ & $\begin{array}{c}0 \\
1(6 \%) \\
11(65 \%)\end{array}$ & $\begin{array}{l}0 \\
0\end{array}$ \\
\hline Widder et $a l^{36}$ & Mean 8.8 yrs & Encapsulated $N=26$ & Without invasion $N=26$ & 0 & 0 & 0 \\
\hline Piana et $a l^{37}$ & Mean 11.9 yrs & Encapsulated $N=66$ & Without invasion $N=45$ & & & 0 \\
\hline Liu et $a l^{38}$ & Mean 6.8 yrs & Encapsulated $N=30$ & Without invasion $N=30$ & 0 & 0 & 0 \\
\hline Vivero et $a l^{6}$ & Median 9.3 yrs & $\begin{array}{l}\text { Encapsulated } N=27 \\
\text { Partially encapsulated/well } \\
\text { circumscribed } N=35 \\
\text { Infiltrative } N=9\end{array}$ & $\begin{array}{l}\text { Without invasion } N=14 \\
\text { With invasion } N=13\end{array}$ & $\begin{array}{c}0 \\
0 \\
0 \\
7(78 \%)\end{array}$ & $\begin{array}{c}0 \\
0 \\
1(3 \%)^{b}\end{array}$ & $\begin{array}{l}0 \\
0\end{array}$ \\
\hline Rosario et al ${ }^{39}$ & Median 6 yrs & Encapsulated $N=57$ & Without invasion $N=57$ & 0 & 0 & 0 \\
\hline Howitt et al ${ }^{40}$ & Median 3 yrs & Encapsulated $N=79$ & Without invasion $N=79$ & 0 & 0 & 0 \\
\hline Ganly et $a l^{41}$ & Median 9.5 yrs & Encapsulated $N=83$ & $\begin{array}{l}\text { Without invasion } N=57 \\
\text { With invasion } N=26\end{array}$ & $\begin{array}{l}0 \\
0\end{array}$ & $\begin{array}{l}0 \\
4\end{array}$ & 0 \\
\hline Nikiforov et $a l^{7}$ & $\begin{array}{l}\text { Median } 13 \text { yrs (encapsulated } \\
\text { non-invasive) }\end{array}$ & Encapsulated $N=210$ & $\begin{array}{l}\text { Without invasion } N=109 \\
\text { With invasion } N=101\end{array}$ & $\begin{array}{c}0 \\
1(1 \%)\end{array}$ & $\begin{array}{c}0 \\
5+5^{\mathrm{c}}(10 \%)\end{array}$ & $\begin{array}{c}0 \\
2(2 \%)\end{array}$ \\
\hline Thompson ${ }^{28}$ & Median 11.8 yrs & Encapsulated $N=94$ & $\begin{array}{l}\text { Without invasion } N=77^{\mathrm{d}} \\
\text { With invasion } N=17\end{array}$ & 0 & 0 & 0 \\
\hline Cho et $a 1^{24}$ & Median 3.1 yrs & Encapsulated $N=152^{\mathrm{e}}$ & $\begin{array}{l}\text { Without invasion } N=105 \\
\text { With invasion } N=47\end{array}$ & $\begin{array}{l}3(3 \%) \\
4(9 \%)\end{array}$ & $\begin{array}{l}1(1 \%) \\
1(2 \%)\end{array}$ & \\
\hline
\end{tabular}

${ }^{\mathrm{a}}$ One patient with questionable noninvasive nature of the tumor

${ }^{\mathrm{b}}$ One patient with positive margin.

${ }^{\mathrm{c}}$ Five patients with detectable thyroglobulin.

$\mathrm{d}_{45}$ of the noninvasive tumors in this study were part of the series from Nikiforov et al. ${ }^{7}$

${ }^{\mathrm{e}}$ Represents the profile of the cohort when $<1 \%$ papillae were used as the cutoff.n/r, not reported. 
Table 3 Summary of grossing and reporting guidelines

Grossing recommendations

- The entire tumor capsule or tumor normal interface is submitted for histologic evaluation

- For large lesions, stepwise submission of sections (ie, a limited number initially) until invasion is found or the lesional border is entirely submitted is acceptable

- Multiple sections can be submitted per block, focusing on the tumor periphery and its junction to the parenchyma

- In the setting of multinodular disease gross identification of a fine needle tract may be beneficial to capture the lesion of interest

- For lesions with excessively overt nuclear features of papillary carcinoma but without exclusion criteria on initial sectioning, additional sections of the central portion should be submitted to exclude a conventional papillary thyroid carcinoma component

\section{Reporting parameters}

- NIFTP does not require a formal staging (ie, no AJCC or UICC stage)

- Certain oncologic parameters are redundant (ie, the absence of vascular invasion)

- A limited data set consisting of tumor size, laterality and margin status may be useful

- Ancillary immunohistochemical markers such as HBME-1 and Galectin-3 should be applied with caution.

- During this current period of transition, a comment linking NIFTP to its prior designation as non-invasive/encapsulated/ well-demarcated papillary thyroid carcinoma is recommended.

nodal disease rather than the NIFTP itself. None of the other well-documented cases of NIFTP metastasized or recurred, even when the only treatment was lobectomy. Therefore, the newly proposed terminology of NIFTP appears to be an appropriate term matching the clinical behavior of this neoplasm. ${ }^{47}$ While clinical managment is evolving, NIFTP would be considered eligible for limited initial surgery (lobectomy) alone, when matching its malignant potential with the most current 2015 American Thyroid Association management guidelines. ${ }^{48}$

\section{Gross examination and reporting}

A summary of gross examination and microscopic reporting recommendations is provided in Table 3. As NIFTP is defined by the absence of tumoral capsular or lymphovascular invasion, thorough examination of the tumor-to-capsule-to-normal interface is critical to ensure appropriate classification. In practical terms, a thorough exam is likely already occurring in most cases, since NIFTP and follicular adenoma will have a similar gross appearance, and as such the gross examiner will likely reflexively sample the tumor normal interface as per institutional protocols. This is simple in the setting of a solitary nodule, but if multiple nodules are present, documentation of nodule location and correlation with the fine needle aspiration should be performed to assure proper sampling of the correct nodule and the tumor-normal interface.

The definition of adequate capsular sampling in NIFTP is controversial and varies by institution and geography. This issue has been previously evaluated in detail over the decades, ${ }^{49-52}$ but has not adequately been studied in a truly prospective or longitudinal manner with outcome correlation determining a reference standard. Nonetheless, we currently advocate the submission of the entire lesional capsule or tumor to normal interface for histologic examination (Figure 10). The mean tumor size of NIFTP is about $3.0 \mathrm{~cm}$ and can often be sampled in its entirety within 20 sections; this would likely equate to fewer cassettes if sectioned to focus on the tumor normal interface as noted in Figure 10. For larger nodules, it may however be reasonable to approach total submission of the lesional capsule in a stepwise fashion as suggested by Thompson et al, ${ }^{50}$ with an initial generous but limited sampling, followed by submission of the remainder of the tumoral capsule if no vascular or capsular invasion is initially detected. Tumors with very prominent nuclear features of papillary thyroid carcinoma, but no clear cut exclusionary features on initial examination, may require additional sections of the central portion of the tumor to exclude a conventional component. In such scenarios, smaller lesions $(3.0 \mathrm{~cm}$ or less) should be entirely submitted. For larger lesions, at least one central section per $\mathrm{cm}$ should be submitted. As this lesion has only recently been reclassified to an indolent category, prospective thorough evaluation ensures the integrity of the gross examination and subsequent microscopic confirmation of its biologic potential.

Reporting of NIFTP will be an evolving process. As it is no longer reported as a 'cancer,' the first implication is that it is no longer subject to the same formal staging as thyroid carcinomas. Additionally, NIFTP will no longer be subject to all the required data elements from the current thyroid College of American Pathologists cancer reporting protocol. Many parameters are only relevant to cancers, and reporting negative findings such as absence of capsular and vascular invasion would be redundant with respect to the NIFTP category, which is defined by their absence. Perhaps the only relevant parameters would be tumor location (laterality), tumor size, and margin status, and documentation of fine needle associated changes, if applicable. This is more akin to what is reported for indolent tumors that still have the potential to recur if incompletely excised (and as reported for a case of recurrent, incompletely excised encapsulated follicular variant of papillary thyroid carcinoma). ${ }^{6}$

At the same time, these tumors are not yet considered entirely benign, hence their current designation. It may still be beneficial to capture these lesions in a codified fashion, possibly as a limited data set in synoptic format. The College of American Pathologists has now included NIFTP as 


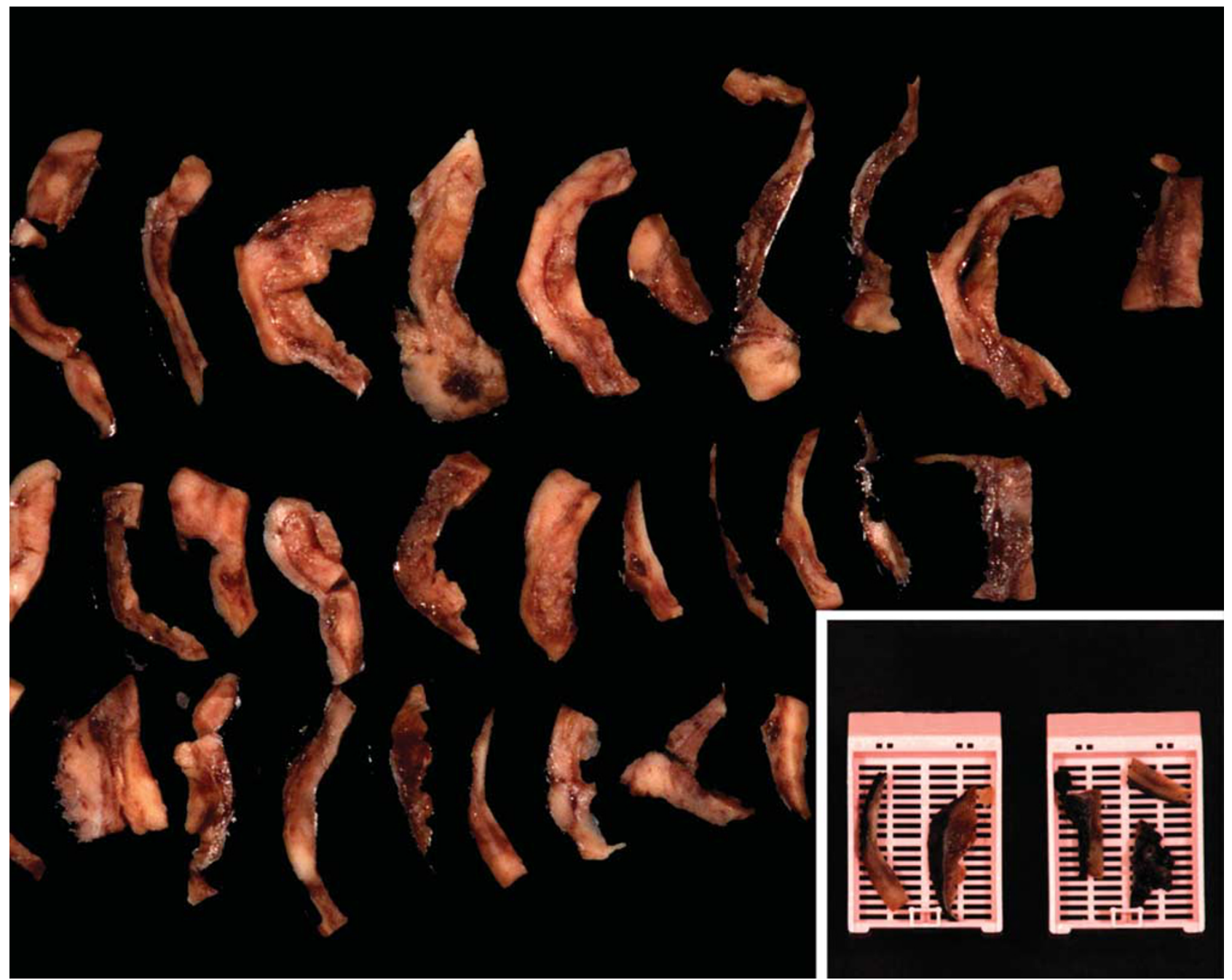

Figure 10 The tumoral capsule of a $5.0 \mathrm{~cm}$ lesion as a rind with a thin rim of normal sectioned in its entirety. Multiple sections of this tumor capsule with the surrounding non neoplastic parenchyma can be submitted in one cassette. With this technique, the lesional interface was submitted within 30 sections; most NIFTP are actually considerably smaller than $5.0 \mathrm{~cm}$.

an optional data element in the latest version of the thyroid protocol, effective $1 / 1 / 2018 .{ }^{53}$ Linked to this approach, would be capturing these tumors in a registry. In most countries, registries are built around capturing cancers, not benign tumors. Thus, the danger here is the potential loss of population based statistical data on this tumor. Potential workarounds in addition to a limited reporting protocol may entail newer ICD-O3 coding and coordination with the National Cancer Registry Association to create a mechanism to capture this category.

Reclassification to NIFTP will also impact use and reporting of ancillary immunohistochemical markers, such as HBME1 and Galectin-3, which may show immunoreactivity in NIFTP. While the role of these remains to be defined, the same technical and interpretive caveats for these markers will still likely remain. ${ }^{38,54,55}$ Regarding the adjunct immunostain, BRAFVE, the BRAF V600E-specific antibody, it will likely see little use in this category, except to exclude a NIFTP diagnosis, since it may help tease out deceptively well-demarcated follicular patterned but classic papillary thyroid carcinoma in some instances. ${ }^{40,56}$ Along these lines, molecular testing may impact the diagnosis in a given case. While NIFTP remains primarily a morphologic diagnosis, when molecular data are available on a given case, the aforementioned molecular alterations more typical of NIFTP would substantiate the diagnosis further, whereas unexpected molecular alterations such as those involving BRAF V600E, TERT, RET/ $P T C$, or $A L K$, are essentially exclusionary and should prompt further investigation for morphologic exclusion criteria. Along these lines, a recent study by Lee et $a^{57}$ claiming a $28.6 \%$ prevalence of $B R A F$ V600E in NIFTP in their cohort should thus be viewed with considerable skepticism as to the strictness of the inclusion and exclusion criteria employed.

Finally, as individual practitioners making the transition to using NIFTP terminology instead of encapsulated follicular variant of papillary thyroid carcinoma, it may be reasonable to bridge this 
transition, both for pathologists and clinicians, by providing an explanatory note at the end of cases. A few points that the note may include are that NIFTP has been determined, under strict criteria, to be of exceptionally low biological potential and that no additional surgery (outside of lobectomy) or treatment is warranted for this tumor, with a deescalation of clinical follow-up recommended.

\section{Limitations and challenges: a starting point... not a final solution}

NIFTP is not a 'finished product.' While it does represent the most current synthesis of morphologic, molecular, and biologic understanding of this category of tumors, it is still evolving and the concepts outlined here require validation and if needed, modification. The most obvious issue at first glance is the name itself. Even for proponents of this concept, the full name, 'noninvasive follicular thyroid neoplasm with papillary-like nuclear features' is admittedly unwieldy. This goes beyond aesthetic linguistic appeal as this terminology may be cumbersome to report, and may be even more awkward when translated to other languages. However, the acronym NIFTP is more palatable, and the term itself aptly characterizes this entity. Other general limitations include the retrospective nature and selection bias in studies, absence of large-scale population level data, absence of neck dissection on many patients to truly establish pNo status, and limited data on extreme long term follow-up (ie, 30 years) often regarded as the optimal measurement for well-differentiated thyroid follicular epithelial cancers that may show very late recurrences. A recent ATA guideline has now accepted this terminology, but has categorized this as a weak recommendation, based on moderate quality evidence, basically echoing the need for additional studies. ${ }^{58}$ Similarly, NIFTP has been included in the upcoming 4th edition of the World Health Organization Classification of Endocrine Tumors.

One critical point is that this nomenclature shift will not rectify all the difficulties of follicular patterned lesions. While it may curtail overdiagnosis of some cancers, it does not directly address the more overarching problem of a binary view on cancer (of all types) that is often the norm in practice settings. The term NIFTP has come into existence as an attempt to reduce one aspect of this issue, but also highlights a fundamental defect in recognizing the variety in biologic behavior in thyroid neoplasia. This requires education and a better means for conveying in a standardized fashion that the spectrum of neoplasia is more graded than 'benign' or 'malignant.'

The prospective diagnosis of NIFTP in the reporting of fine needle aspiration findings is another area of transition. While 'The Bethesda System for Reporting of Thyroid Fine Needle Aspiration
Cytology' has six categories with graded risks of malignancy, ${ }^{59}$ endpoints are binary (ie, cancer or no cancer). As the thyroid Bethesda reporting system has its basis in nuclear features detected cytologically, NIFTP, with its nuclear features of papillary thyroid carcinoma has the potential to increase falsepositive results, if not accounted for by modification of the risks of malignancy for each category. It would seem that the risk of malignancy will decrease in each category, but perhaps less so in the malignant category ${ }^{60-63}$ Cytologic criteria that would favor NIFTP/follicular variant of papillary thyroid carcinoma over conventional papillary thyroid carcinoma have recently been proposed, and may influence reporting as well. ${ }^{64}$

NIFTP allows for a 'cushion' between clearly benign and overtly malignant tumors which to some extent eases the diagnostic challenge when encountering an encapsulated lesion with only moderately developed nuclear features. A general concern for such a category is potential abuse as a 'wastebasket' category. The risk for the development of this phenomenon is difficult to predict but efforts must be made to continue to apply the aforementioned inclusion and exclusion criteria to mitigate this effect. To this end, the nuclear scoring scheme may allow for a more systematic approach to classification, but does not entirely remove the issues of interobserver variability. Furthermore, while nuclear scoring was well tested in the defining multiinstitution study, ${ }^{7}$ other criteria such as infiltration, lymphovascular invasion, and what qualifies as true papillary growth were not actually well vetted by the expert panel, leaving room for refinement in future studies.

Some issues that were not addressed include applicability to predominantly oncocytic follicular patterned lesions, multifocal lesions, and tumors $<1.0 \mathrm{~cm}$. Conceptually, NIFTP seems plausible in these groups but validation studies will be necessary. Thus the diagnosis is not contraindicated in these situations, but it is not yet well vetted. NIFTP was successfully applied to a few multifocal, and a few sub centimeter tumors. ${ }^{28}$ Of note, the United Kingdom Royal College of Pathologists has already listed oncocytic morphology as an exclusionary criterion. ${ }^{65}$ While there may be differences in using molecular profile as an endpoint, and while various parameters, notably nuclear features (since oncocytic nuclei are enlarged and often show membrane irregularities), might be different, the working group has not yet excluded the possibility of an oncocytic NIFTP.

\section{Conclusions}

The diagnostic category of NIFTP prevents overtreatment of a group of low-risk thyroid tumors formerly known as noninvasive follicular variant papillary thyroid carcinoma. While many histologic 
criteria are quite familiar, NIFTP also carries a stricter set of exclusionary criteria to ensure that this is a homogenous, indolent group. Simplified nuclear scoring may be beneficial to standardize assessment of diagnostic nuclear features. Recurrences and metastases are exceptionally rare, and rigorous application of the delineated criteria will ensure this. In order to ensure that there is no invasion, total submission of the tumor normal interface is advocated. The framework for reporting of NIFTP in a standardized fashion is still evolving, but will no longer be the same as for papillary thyroid carcinoma. Several conceptual and morphologic challenges and limitations remain signifying that NIFTP is only an initial step towards a more biologically accurate approach to thyroid neoplasia.

\section{Disclosure/conflict of interest}

The authors declare no conflicts of interest.

\section{References}

1 Davies L, Welch HG. Increasing incidence of thyroid cancer in the United States, 1973-2002. JAMA 2006;295:2164-2167.

2 Jung CK, Little MP, Lubin JH, et al. The increase in thyroid cancer incidence during the last four decades is accompanied by a high frequency of BRAF mutations and a sharp increase in RAS mutations. J Clin Endocrinol Metab 2014;99:E276-E285.

3 Elsheikh TM, Asa SL, Chan JK, et al. Interobserver and intraobserver variation among experts in the diagnosis of thyroid follicular lesions with borderline nuclear features of papillary carcinoma. Am J Clin Pathol 2008;130:736-744.

4 Liu J, Singh B, Tallini G, et al. Follicular variant of papillary thyroid carcinoma: a clinicopathologic study of a problematic entity. Cancer 2006;107:1255-1264.

5 Rivera M, Ricarte-Filho J, Knauf J, et al. Molecular genotyping of papillary thyroid carcinoma follicular variant according to its histological subtypes (encapsulated vs infiltrative) reveals distinct BRAF and RAS mutation patterns. Mod Pathol 2010;23:1191-1200.

6 Vivero M, Kraft S, Barletta JA. Risk stratification of follicular variant of papillary thyroid carcinoma. Thyroid 2013;23:273-279.

7 Nikiforov YE, Seethala RR, Tallini G, et al. Nomenclature revision for encapsulated follicular variant of papillary thyroid carcinoma: a paradigm shift to reduce overtreatment of indolent tumors. JAMA Oncol 2016;2: 1023-1029.

8 Meissner W, Warren S (eds). Tumors of the thyroid gland. Atlas of Tumor Pathology (2nd series, Fascicle 4). Armed Forces Institute of Pathology: Washington, DC, 1969, pp 70-92.

9 Lindsay S. Carcinoma of the Thyroid Gland: A Clinical and Pathologic Study of 239 Patients at the University of California Hospital. Charles C Thomas: Springfield, IL, 1960.

10 Chen K, Rosai J. Follicular variant of thyroid papillary carcinoma: A clinicopathologic study of six cases. Am J Surg Pathol 1977;1:123-130.
11 LiVolsi VA, Albores-Saavedra J, Asa SL, et al. Papillary carcinoma. In: DeLellis RA, Lloyd RD, Heitz PU, et al. (eds). Pathology and Genetics. Tumours of Endocrine Organs. World Health Organization Classification of Tumours, 3rd edn. IARC Press: Lyon, France, 2004, pp 57-66.

12 Rosai J, Carcangiu M, DeLellis. Papillary carcinoma. In: Rosai J, Carcangiu M, DeLellis (eds). Tumors of the Thyroid Gland. Atlas of Tumor Pathology (3rd series, Fascicle 5). Armed Forces Institute of Pathology: Washington DC, 1993, pp 65-122.

13 Lloyd RV, Erickson LA, Casey MB, et al. Observer variation in the diagnosis of follicular variant of papillary thyroid carcinoma. Am J Surg Pathol 2004;28: 1336-1340.

14 Hirokawa M, Carney JA, Goellner JR, et al. Observer variation of encapsulated follicular lesions of the thyroid gland. Am J Surg Pathol 2002;26:1508-1514.

15 LiVolsi VA, Merino MJ. Worrisome histologic alterations following fine-needle aspiration of the thyroid (WHAFFT). Pathol Annu 1994;29(Pt 2):99-120.

16 Papotti M, Rodriguez J, De Pompa R, et al. Galectin-3 and HBME-1 expression in well-differentiated thyroid tumors with follicular architecture of uncertain malignant potential. Mod Pathol 2005;18:541-546.

17 Baloch ZW, LiVolsi VA. Our approach to follicularpatterned lesions of the thyroid. J Clin Pathol 2007;60: 244-250.

18 Williams ED. Guest editorial: two proposals regarding the terminology of thyroid tumors. Int J Surg Pathol 2000;8:181-183.

19 Kakudo K, Wasaka T, Kakudo M, et al. Borderline and precursor lesions of thyroid neoplasms: a missing link. J Basic Clin Med 2015;4:2-7.

20 Esserman LJ, Thompson IM, Reid B, et al. Addressing overdiagnosis and overtreatment in cancer: a prescription for change. Lancet Oncol 2014;15:e234-e242.

21 Chan J. Strict criteria should be applied in the diagnosis of encapsulated follicular variant of papillary thyroid carcinoma. Am J Clin Pathol 2002;117:16-18.

22 Mete O, Asa SL. Pathological definition and clinical significance of vascular invasion in thyroid carcinomas of follicular epithelial derivation. Mod Pathol 2011;24: 1545-1552.

23 Hiltzik D, Carlson DL, Tuttle RM, et al. Poorly differentiated thyroid carcinomas defined on the basis of mitosis and necrosis: a clinicopathologic study of 58 patients. Cancer 2006;106:1286-1295.

24 Cho U, Mete O, Kim MH, et al. Molecular correlates and rate of lymph node metastasis of non-invasive follicular thyroid neoplasm with papillary-like nuclear features and invasive follicular variant papillary thyroid carcinoma: the impact of rigid criteria to distinguish non-invasive follicular thyroid neoplasm with papillary-like nuclear features. Mod Pathol 2017;30: 810-825.

25 Johannessen JV, Sobrinho-Simoes M. The origin and significance of thyroid psammoma bodies. Lab Invest 1980;43:287-296.

26 Bongiovanni M, Mazzucchelli L, Giovanella L, et al. Well-differentiated follicular patterned tumors of the thyroid with high-grade features can metastasize in the absence of capsular or vascular invasion: report of a case. Int J Surg Pathol 2014;22:749-756.

27 Perez-Montiel MD, Suster S. The spectrum of histologic changes in thyroid hyperplasia: a clinicopathologic study of 300 cases. Hum Pathol 2008;39:1080-1087. 
28 Thompson LD. Ninety-four cases of encapsulated follicular variant of papillary thyroid carcinoma: a name change to noninvasive follicular thyroid neoplasm with papillary-like nuclear features would help prevent overtreatment. Mod Pathol 2016;29:698-707.

29 Rosai J, Zampi G, Carcangiu ML. Papillary carcinoma of the thyroid. A discussion of its several morphologic expressions, with particular emphasis on the follicular variant. Am J Surg Pathol 1983;7:809-817.

30 Renshaw AA, Gould EW. Why there is the tendency to 'overdiagnose' the follicular variant of papillary thyroid carcinoma. Am J Clin Pathol 2002;117:19-21.

31 Baloch ZW, Livolsi VA. Follicular-patterned lesions of the thyroid: the bane of the pathologist. Am J Clin Pathol 2002;117:143-150.

32 Hunt JL, Dacic S, Barnes EL, et al. Encapsulated follicular variant of papillary thyroid carcinoma. Am J Clin Pathol 2002;118:602-603 author reply 5-6.

33 Nikiforov YE, Carty SE, Chiosea SI, et al. Highly accurate diagnosis of cancer in thyroid nodules with follicular neoplasm/suspicious for a follicular neoplasm cytology by ThyroSeq v2 next-generation sequencing assay. Cancer 2014;120:3627-3634.

34 Vanzati A, Mercalli F, Rosai J. The 'sprinkling' sign in the follicular variant of papillary thyroid carcinoma: a clue to the recognition of this entity. Arch Pathol Lab Med 2013;137:1707-1709.

35 Baloch ZW, LiVolsi VA. Encapsulated follicular variant of papillary thyroid carcinoma with bone metastases. Mod Pathol 2000;13:861-865.

36 Widder S, Guggisberg K, Khalil M, et al. A pathologic re-review of follicular thyroid neoplasms: the impact of changing the threshold for the diagnosis of the follicular variant of papillary thyroid carcinoma. Surgery 2008;144:80-85.

37 Piana S, Frasoldati A, Di Felice E, et al. Encapsulated well-differentiated follicular-patterned thyroid carcinomas do not play a significant role in the fatality rates from thyroid carcinoma. Am J Surg Pathol 2010;34: 868-872.

38 Liu Z, Zhou G, Nakamura M, et al. Encapsulated follicular thyroid tumor with equivocal nuclear changes, so-called well-differentiated tumor of uncertain malignant potential: a morphological, immunohistochemical, and molecular appraisal. Cancer Sci 2011;102:288-294.

39 Rosario PW, Penna GC, Calsolari MR. Noninvasive encapsulated follicular variant of papillary thyroid carcinoma: is lobectomy sufficient for tumours $>$ / $=1 \mathrm{~cm}$ ? Clin Endocrinol 2014;81:630-632.

40 Howitt BE, Paulson VA, Barletta JA. Absence of BRAF V600E in non-infiltrative, non-invasive follicular variant of papillary thyroid carcinoma. Histopathology 2015;67:579-582.

41 Ganly I, Wang L, Tuttle RM, et al. Invasion rather than nuclear features correlates with outcome in encapsulated follicular tumors: further evidence for the reclassification of the encapsulated papillary thyroid carcinoma follicular variant. Hum Pathol 2015;46: 657-664

42 Niemeier LA, Kuffner Akatsu H, Song C, et al. A combined molecular-pathologic score improves risk stratification of thyroid papillary microcarcinoma. Cancer 2012;118:2069-2077.

43 Kakudo K, Bai Y, Liu Z, et al. Classification of thyroid follicular cell tumors: with special reference to borderline lesions. Endocr J 2012;59:1-12.
44 Ito Y, Yabuta T, Hirokawa M, et al. Distant and lymph node metastases of thyroid nodules with no pathological evidence of malignancy: a limitation of pathological examination. Endocr J 2008;55:889-894.

45 Jiang XS, Harrison GP, Datto MB. Young Investigator Challenge: molecular testing in noninvasive follicular thyroid neoplasm with papillary-like nuclear features. Cancer 2016;124:893-900.

46 Jiang XS, Harrison GP, Datto MB. Reply to Young Investigator Challenge: molecular testing in noninvasive follicular thyroid neoplasm with papillary-like nuclear features. Cancer 2017;125:293-294.

47 Hodak S, Tuttle RM, Maytal G, et al. Changing the cancer diagnosis: the case of follicular variant of papillary thyroid cancer-primum non nocere and NIFTP. Thyroid 2016;26:869-871.

48 Haugen BR, Alexander EK, Bible KC, et al. 2015 American Thyroid Association management guidelines for adult patients with thyroid nodules and differentiated thyroid cancer: the American Thyroid Association Guidelines Task Force on Thyroid Nodules and Differentiated Thyroid Cancer. Thyroid 2016;26: 1-133.

49 Yamashina M. Follicular neoplasms of the thyroid. Total circumferential evaluation of the fibrous capsule. Am J Surg Pathol 1992;16:392-400.

50 Thompson LD, Wieneke JA, Paal E, et al. A clinicopathologic study of minimally invasive follicular carcinoma of the thyroid gland with a review of the English literature. Cancer 2001;91:505-524.

51 Hazard JB, Kenyon R. Atypical adenoma of the thyroid. AMA Arch Pathol 1954;58:554-563.

52 Lang W, Georgii A, Stauch G, et al. The differentiation of atypical adenomas and encapsulated follicular carcinomas in the thyroid gland. Virchows Arch A Pathol Anat Histol 1980;385:125-141.

53 Seethala RR, Asa SL, Bullock MB, et al. Protocol for the examination of specimens from patients with carcinomas of the thyroid gland 2017, Available at http:// www.cap.org/ShowProperty?nodePath =/UCMCon/ Contribution\% 20Folders/WebContent/pdf/cp-thyroid17protocol-4000.pdf.

54 Wallander M, Layfield LJ, Jarboe E, et al. Follicular variant of papillary carcinoma: reproducibility of histologic diagnosis and utility of HBME-1 immunohistochemistry and BRAF mutational analysis as diagnostic adjuncts. Appl Immunohistochem Mol Morphol 2010;18:231-235.

55 Gucer H, Bagci P, Bedir R, et al. The value of HBME-1 and claudin-1 expression profile in the distinction of BRAF-like and RAS-like phenotypes in papillary thyroid carcinoma. Endocr Pathol 2016;27: 224-232.

56 Routhier CA, Mochel MC, Lynch K, et al. Comparison of 2 monoclonal antibodies for immunohistochemical detection of BRAF V600E mutation in malignant melanoma, pulmonary carcinoma, gastrointestinal carcinoma, thyroid carcinoma, and gliomas. Hum Pathol 2013;44:2563-2570.

57 Lee SE, Hwang TS, Choi YL, et al. Molecular profiling of papillary thyroid carcinoma in Korea with a high prevalence of BRAFV600E mutation. Thyroid 2017;27: 802-810.

58 Haugen BRM, Sawka AM, Alexander EK, et al. The ATA Guidelines on Management of Thyroid Nodules and Differentiated Thyroid Cancer Task Force Review and recommendation on the proposed renaming of 
eFVPTC without invasion to NIFTP. Thyroid 2017;27: 481-483.

59 Ali SZ. Thyroid cytopathology: Bethesda and beyond. Acta Cytol 2011;55:4-12.

60 Baloch ZW, Seethala RR, Faquin WC, et al. Noninvasive follicular thyroid neoplasm with papillary-like nuclear features (NIFTP): a changing paradigm in thyroid surgical pathology and implications for thyroid cytopathology. Cancer Cytopathol 2016;124:616-620.

61 Maletta F, Massa F, Torregrossa L et al. Cytological features of "non-invasive follicular thyroid neoplasm with papillary-like nuclear features" and their correlation with tumor histology. Hum Pathol 2016;54:134-142.

62 Strickland KC, Howitt BE, Marqusee E, et al. The impact of noninvasive follicular variant of papillary thyroid carcinoma on rates of malignancy for fine-needle aspiration diagnostic categories. Thyroid 2015;25:987-992.

63 Howitt BE, Chang S, Eszlinger M, et al. Fine-needle aspiration diagnoses of noninvasive follicular variant of papillary thyroid carcinoma. Am J Clin Pathol 2015;144:850-857.

64 Strickland KC, Vivero M, Jo VY, et al. Preoperative cytologic diagnosis of noninvasive follicular thyroid neoplasm with papillary-like nuclear features: a prospective analysis. Thyroid 2016;26:1466-1471.

65 Johnson S, Stephenson TJ, Poller DN, NIFTP addendum to the RCPath dataset for thyroid cancer histopathology reports 2016, Available at https://www. rcpath.org/resourceLibrary/thyroiddataset-niftpadden dum-jun16-pdf.html. 